\title{
A Patient-Derived Orthotopic Xenograft Model of Gastroesophageal-Junction Adenocarcinoma Translated to the Clinic by Tumor-Targeting Fluorescent Antibodies to Carcinoembryonic-Antigen-Related Cell-Adhesion Molecules
}

\author{
MICHAEL A. TURNER ${ }^{1,2}$, SIAMAK AMIRFAKHRI ${ }^{1,2}$, HIROTO NISHINO $^{1,2}$, \\ THINZAR M. LWIN ${ }^{1}$, THOMAS J. SAVIDES ${ }^{3}$, TONY R. REID ${ }^{3}$, \\ BERNHARD B. SINGER ${ }^{4}$, ROBERT M. HOFFMAN ${ }^{1,2,5}$ and MICHAEL BOUVET ${ }^{1,2}$ \\ ${ }^{1}$ Department of Surgery, University of California San Diego, San Diego, CA, U.S.A.; \\ ${ }^{2}$ VA San Diego Healthcare System, San Diego, CA, U.S.A.; \\ ${ }^{3}$ Department of Medicine, University of California San Diego, San Diego, CA, U.S.A.; \\ ${ }^{4}$ Institute of Anatomy, Medical Faculty, University of Duisburg-Essen, Essen, Germany; \\ ${ }^{5}$ AntiCancer, Inc., San Diego, CA, U.S.A.
}

\begin{abstract}
Background/Aim: During surgical resection of gastroesophageal-junction (GEJ) adenocarcinoma, the margin status is often difficult to visualize resulting in high recurrence rates. The aim of the present study was to develop a labelling technique that would allow improved visualization of GEJ tumor margins for surgeons to reduce recurrence rates in a patient-like model. Materials and Methods: A patient GEJ tumor was obtained from an endoscopic biopsy and implanted subcutaneously in a nude mouse. A patientderived orthotopic xenograft (PDOX) model was established by implanting tumor fragments grown from a subcutaneous model to the cardia of the stomach of nude mice. CC1/3/5$S A B$, an antibody to carcinoembryonic-antigen-related celladhesion molecules, was conjugated with infrared dye IRDye800 to create SAB-IR800. Forty-eight hours after i.v. injection of SAB-IR800, GEJ-PDOX mice were imaged. Results: Fluorescence imaging with SAB-IR800 brightly visualized the GEJ adenocarcinoma demonstrating specific targeting. In the PDOX model, injection of SAB-IR800 (50
\end{abstract}

This article is freely accessible online.

Correspondence to: Michael Bouvet, MD, University of California, San Diego, Moores Cancer Center, 3855 Health Sciences Drive \#0987, La Jolla, CA 92093-0987, U.S.A. Tel: +1 8588226191, e-mail: mbouvet@ucsd.edu

Key Words: PDOX, patient-derived orthotopic xenograft, gastroesophageal junction, adenocarcinoma, carcinoembryonic-antigenrelated cell adhesion, fluorescent antibodies. $\mu \mathrm{g})$ resulted in a tumor to background ratio of 1.78 at 48 hours and 1.86 at 72 hours. Conclusion: PDOX models of GEJ tumors can be established from patients by endoscopic biopsy without undergoing surgical resection. GEJ PDOX models should be useful for developing novel diagnostics and therapeutics for this recalcitrant disease.

Gastroesophageal junction (GEJ) tumors are defined as tumors whose origins are within $5 \mathrm{~cm}$ of the anatomical GEJ (1). These tumors have been increasing in incidence in the Western World $(1,2)$. Currently, the only curative treatment for GEJ tumors is surgical resection (3). However, the majority of patients present with advanced disease, and less than 50\% undergo surgery (3). There remains controversy regarding classification and treatment of this type of cancer with wide variations in operative management $(2,3)$. One challenge of surgical resection of GEJ tumors is achieving an $\mathrm{R} 0$ resection which is an independent predictor of survival (1). This present report describes an imaging PDOX model of adenocarcinoma of the GEJ.

Carcinoembryonic-antigen-related cell-adhesion molecules (CEACAMs) are a family of cell-surface glycoproteins. CEACAMs have many cellular functions including cell-cell recognition, cell adhesion, intra- and intercellular signaling as well as functions involved in inflammation, cancer progression and metastasis $(4,5)$. Several CEACAMs have been validated as biomarkers for melanoma, colorectal cancer, lung and pancreatic cancer (4). Specifically CEACAM1 and CEACAM5 are overexpressed in several cancers including gastric cancer (4). $\mathrm{CC} 1 / 3 / 5-\mathrm{SAB}$ is an antibody which selectively binds to human CEACAM1, CEACAM3 and CEACAM5. 

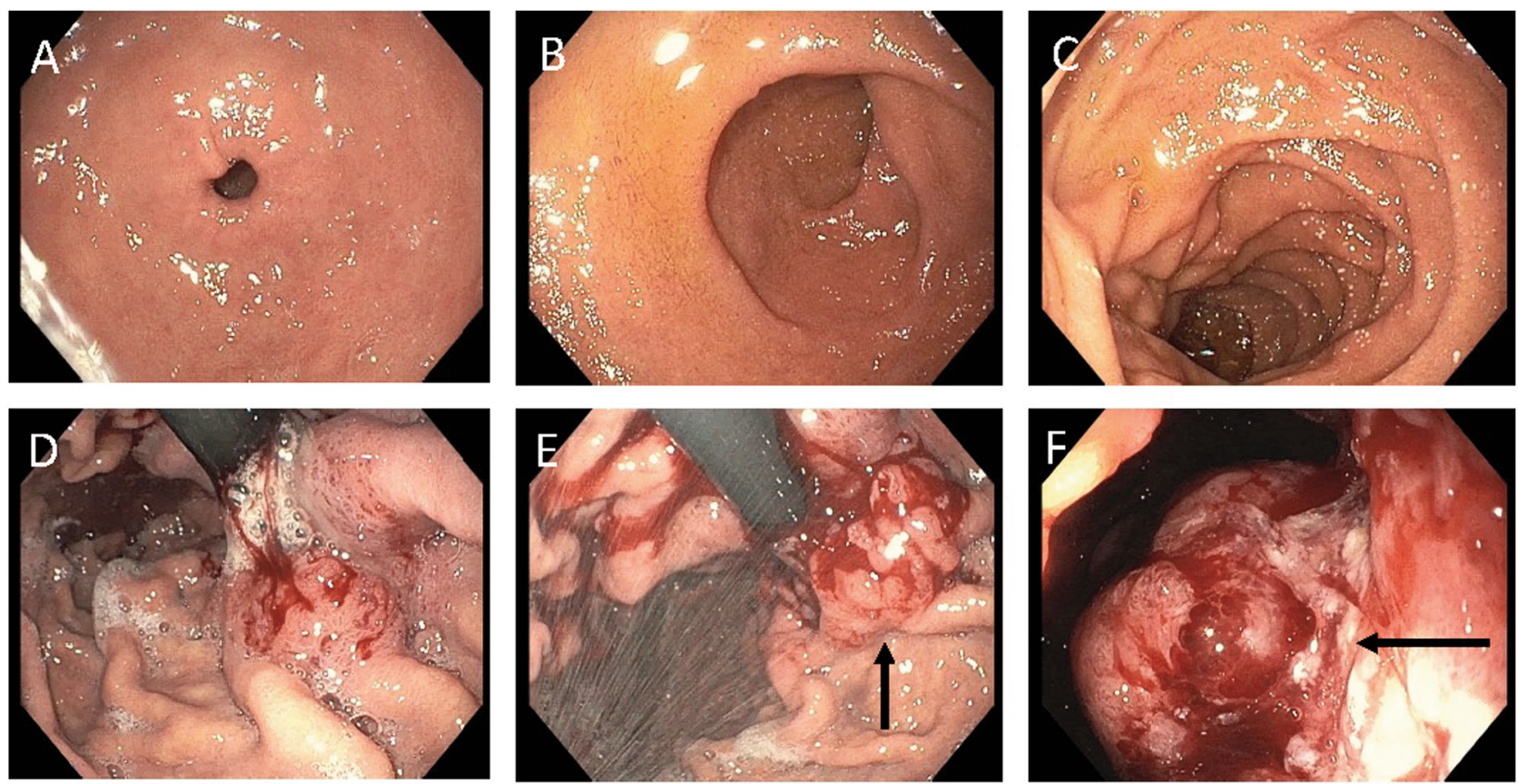

Figure 1. Endoscopic images from a GEJ patient. Panel A shows normal-appearing duodenum. Panels B and C show normal gastric rugae. Panels $D, E$ and $F$ show the endoscope retroflexed to demonstrate a near obstructing gastric cardia mass (black arrows).

In the present study, we used the $\mathrm{CC} 1 / 3 / 5-\mathrm{SAB}$ antibody conjugated to a near infrared $800 \mathrm{~nm}$ dye (SAB-IR800) to brightly label the PDOX model of GEJ adenocarcinoma.

\section{Materials and Methods}

Animal care. Athymic nude mice (Jackson Laboratory, Bar Harbor, $\mathrm{ME}$ ) were maintained in a barrier facility with high-efficiency particulate air (HEPA) filtration and fed an autoclaved laboratory diet. All mice were anesthetized with intraperitoneal ketamine and xylazine reconstituted in phosphate-buffered saline (PBS) prior to surgical procedures. All mice post-laparotomy received buprenorphine subcutaneously the day of the procedure and when displaying signs of distress, for pain control. Mice were euthanized by $\mathrm{CO}_{2}$ inhalation and cervical dislocation at the conclusion of the study. All studies were approved by the San Diego Veterans Administration Medical Center Institutional Animal Care and Use Committee (IACUC, animal use protocol A17-020).

GEJ-PDOX establishment. The patient-derived GEJ-tumor specimen was obtained under standard sterile conditions via diagnostic esophagogastroduodenoscopy (Figure 1). Patient tumors and normal tissue were obtained with informed consent under the UCSD Institutional Review Board (IRB). To initially establish the patient tumor in nude mice, tumor fragments $\left(1 \mathrm{~mm}^{3}\right)$ were implanted over the bilateral upper and lower flanks and allowed to grow to approximately $5 \mathrm{~mm}$ in diameter. To establish a GEJ patient-derived orthotopic xenograft (PDOX) model, mice were anesthetized as described above and the stomach was delivered through a midline ventral incision. A $1 \mathrm{~mm}^{3}$ tumor fragment was sutured to the stomach's lesser curve by the cardia using 8-0 nylon sutures (6). The peritoneum and skin were closed using 6-0 vicryl sutures. Tumor size was initially estimated via palpation.

Imaging. In vivo imaging was performed using the Pearl Trilogy Small Animal Imaging System (LI-COR, Lincoln, NE). The GEJ PDOX models were administered SAB-IR800 $(50 \mu \mathrm{g})$ via tail vein injection. The PDOX models were anesthetized at 48 hours after dye injection and a small ventral incision was made to expose both the stomach and tumor which were imaged using the Pearl Trilogy Small Animal Imaging System. The incision was closed with a 60 vicryl suture. PDOX models were euthanized at 72 hours after dye injection and then a laparotomy was performed. Normal stomach was used as background to obtain a tumor to background ratio (TBR).

Statistical analysis. Statistical analysis was performed with R software (Free Software Foundation, Boston, MA, USA).

\section{Results}

The GEJ PDOX model mimicked the clinical disease. A representative mouse treated with SAB-IR 800 was imaged under brightfield and NIR fluorescence using the Pearl Imager (Figure 2). The bright fluorescence labelling of the tumor was evident. For the PDOX models ( $\mathrm{n}=3)$, the mean tumor signal at 48 hours after dye injection was $0.244( \pm 0.036)$. The TBR at 48 hours ranged from 1.45 to 2.18 with a mean TBR of $1.78( \pm 0.37)$. At 

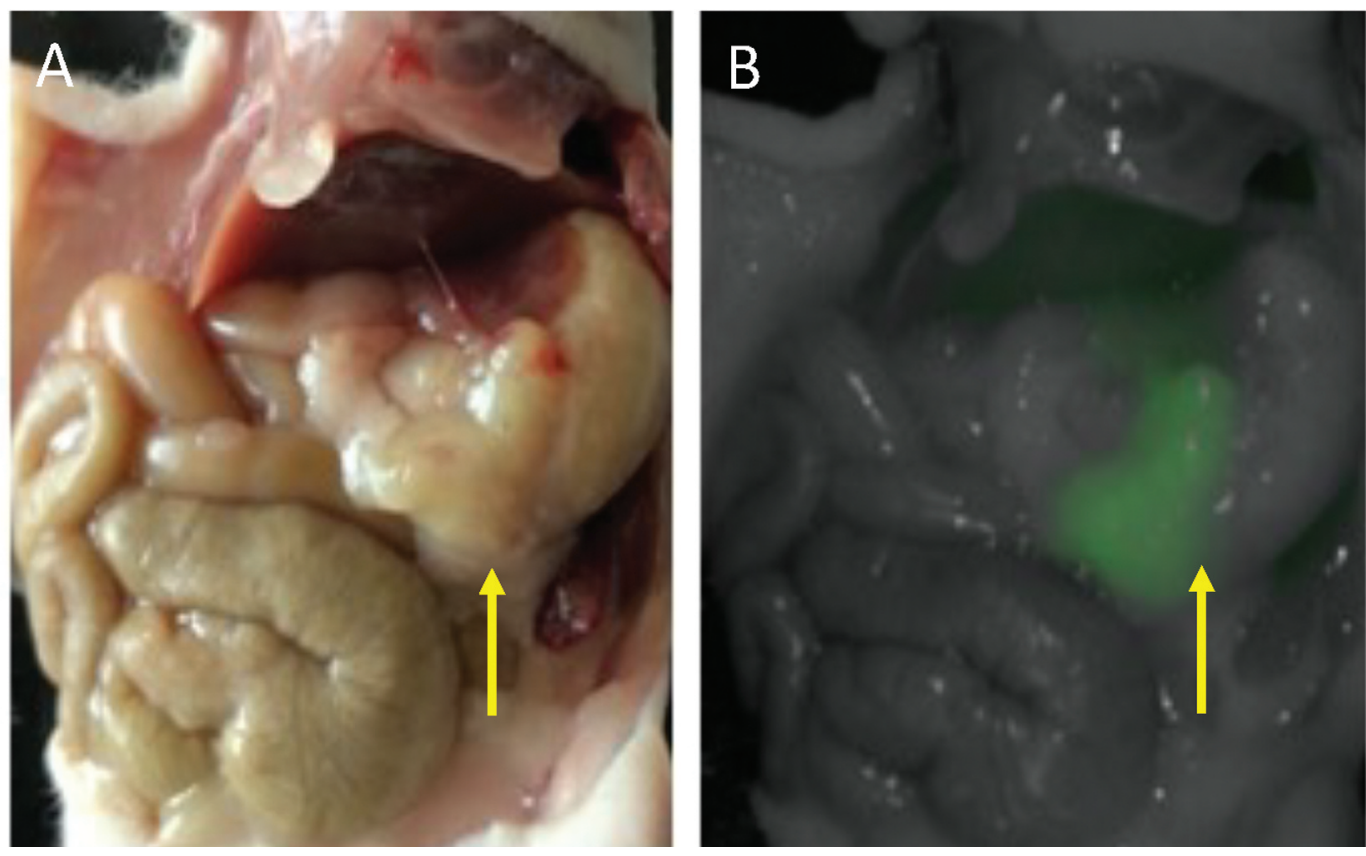

Figure 2. Laparotomy of a representative GEJ-PDOX mouse 72 hours after receiving SAB-IR800 (50 $\mu \mathrm{g})$. Panel A is a brightfield image and Panel $B$ is a fluorescence image. The yellow arrows indicate the patient-derived GEJ tumor growing over the mouse's stomach. Fluorescence labeling with SAB-IR800 resulted in a tumor to background ratio (TBR) of 2.04 .

2.5

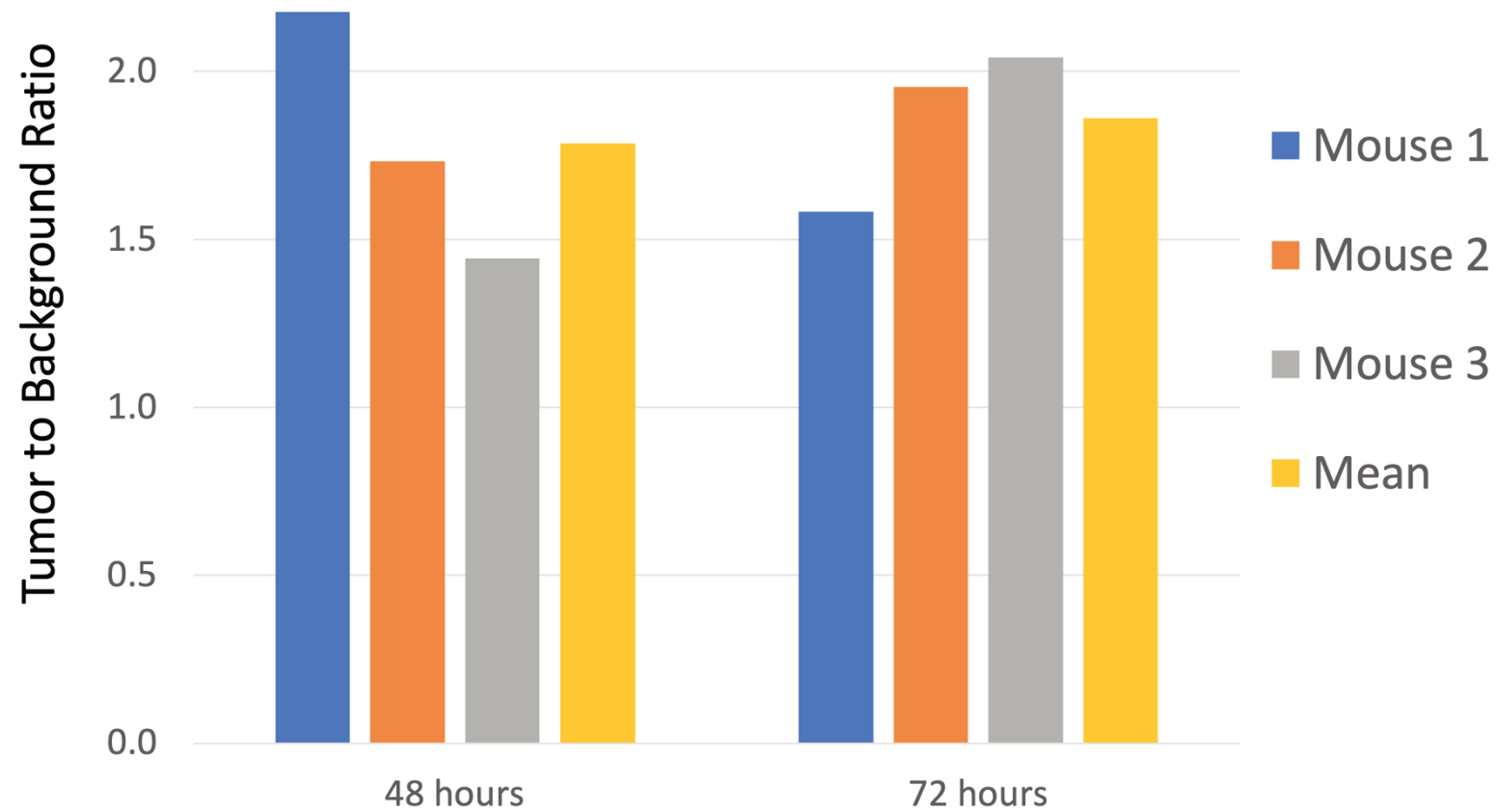

Figure 3. Bar graphs represent the tumor to background ratios (TBRs) of the GEJ PDOX mouse models ( $n=3)$ as well as the mean TBR (yellow bar). 
72 hours, the mean tumor signal was $0.317( \pm 0.075)$. The mean TBR at 72 hours was $1.86( \pm 0.24)$ (Figure 3$)$.

\section{Discussion}

A previous study described a PDOX model of a GEJ tumor (7). However, the present study is the first study to report a GEJ-PDOX mouse model that was successfully imaged with a tumor-specific antibody conjugated to a near-infrared (NIR) fluorophore. Importantly, endoscopically-obtained tissue was sufficient to establish the GEJ tumor in nude mice, thus avoiding a surgical procedure and all the risks inherent with it. The study demonstrated the ability of SAB-IR800 to image GEJ tumors with resultant TBRs that should be sufficient for fluorescence-guided surgery (FGS) in future experiments.

The patient in the present study had a Siewert Type III GEJ tumor originating $>2 \mathrm{~cm}$ below the GEJ. de Graaf et al. reported a series of 416 patients thought to have potentially resectable GEJ tumors (8). After undergoing staging laparoscopy (SL), $84(20.2 \%)$ patients were deemed not surgical candidates, thereby sparing them an unnecessary laparotomy. If some patients have metastatic deposits too small to detect on imaging or visual inspection with SL, FGS could aid the surgeon using the technology described in the present study.

It is difficult to determine the frequency of $\mathrm{R} 0$ resections as some series combine GEJ tumors with esophageal tumors or with gastric adenocarcinoma (2). Along with nodal status and tumor infiltration depth, an R0 resection is an independent predictor of survival (1). We expect SAB-IR800 to become clinically useful for both SL and FGS. Using CEACAMs as an appropriate tumor-targeting agent for FGS is consistent with our previous results with CEACAMs to image colorectal cancer (9).

There are potential indications to use fluorescently-labeled tumors to aid in screening. Iseki et al. demonstrated the effectiveness of using an NIR endoscope to accurately assess gastric-tumor depth to aid in endoscopic mucosal resection (10). Habibollahi et al. used a protease-activatable NIR fluorescent probe to improve detection of human esophageal adenocarcinoma in orthotopic mouse models (11). Tjalma et al. demonstrated that NIR with fluorescent tracers targeting VEGF-A and EGFR improved colorectal-polyp detection over bright light alone, continuing to demonstrate the validity of the principle of the present report in a different organ (12).

The SAB-IR800 has several benefits described in the present report, including high specificity for a GEJ tumor in the PDOX model and a relatively high TBR with potential as a GEJ diagnostic adjunct in SL and FGS. This may lead to improved rate of $\mathrm{R} 0$ resections and patient outcomes. A weakness is this antibody is not a genetic probe (e.g., telomerase-dependent adenovirus expressing GFP) which can genetically label tumors for FGS, as well as recurrence (13-16).
Future studies will be performed with a larger sample size to better characterize the binding and clearing of the SABIR800-dye conjugate to obtain higher TBRs.

\section{Conflicts of Interest}

$\mathrm{RMH}$ is a non-salaried affiliate of AntiCancer, Inc which uses PDOX models for contract research.

\section{Authors' Contributions}

M.A.T., R.M.H. and M.B. designed the experiments and wrote the paper. M.A.T., S.A., H.N., T.M.L., T.J.S., T.R.R., and B.B.S., were involved in the acquisition of the data. All Authors were involved in final manuscript editing and approval.

\section{Acknowledgements}

This study was funded by VA Merit Review grant numbers 1 I01 BX003856-01A1 and 1 I01 BX004494-01 (MB) and NIH/NCI T32CA121938 (MAT and TML).

\section{References}

1 Parry K, Haverkamp L, Bruijnen RC, Siersema PD, Ruurda JP and van Hillegersberg R: Surgical treatment of adenocarcinomas of the gastro-esophageal junction. Ann Surg Oncol 22(2): 597603, 2015. PMID: 25190126. DOI: 10.1245/s10434-014-4047-1

2 Rusch VW: Are cancers of the esophagus, gastroesophageal junction, and cardia one disease, two, or several? Semin Oncol 31(4): 444-449, 2004. PMID: 15297937. DOI: 10.1053/ j.seminoncol.2004.04.023

3 Barbour AP, Rizk NP, Gonen M, Tang L, Bains MS, Rusch VW, Coit DG and Brennan MF: Adenocarcinoma of the gastroesophageal junction: influence of esophageal resection margin and operative approach on outcome. Ann Surg 246(1): 1-8, 2007. PMID: 17592282. DOI: 10.1097/01.sla.0000255563.65157.d2

4 Beauchemin N and Arabzadeh A: Carcinoembryonic antigenrelated cell adhesion molecules (CEACAMs) in cancer progression and metastasis. Cancer Metastasis Rev 32(3-4): 643671, 2013. PMID: 23903773. DOI: 10.1007/s10555-013-9444-6

5 Kuespert K, Pils S and Hauck CR: CEACAMs: their role in physiology and pathophysiology. Curr Opin Cell Biol 18(5): 565571, 2006. PMID: 16919437. DOI: 10.1016/j.ceb.2006.08.008

6 Furukawa T, Fu X, Kubota T, Watanabe M, Kitajima M and Hoffman RM: Nude mouse metastatic models of human stomach cancer constructed using orthotopic implantation of histologically intact tissue. Cancer Res 53(5):1204-1208, 1993. PMID: 8439965.

7 Veeranki OL, Tong Z, Mejia A, Verma A, Katkhuda R, Bassett R, Kim TB, Wang J, Lang W, Mino B, Solis L, Kingsley C, Norton W, Tailor R, Wu JY, Krishnan S, Lin SH, Blum M, Hofstetter W, Ajani J, Kopetz S and Maru D: A novel patient-derived orthotopic xenograft model of esophageal adenocarcinoma provides a platform for translational discoveries. Dis Model Mech 12(12): dmm041004, 2019. PMID: 31732509. DOI: 10.1242/dmm.041004

8 de Graaf GW, Ayantunde AA, Parsons SL, Duffy JP and Welch NT: The role of staging laparoscopy in oesophagogastric cancers. Eur J Surg Oncol 33(8): 988-992, 2007. PMID: 17344017. DOI: $10.1016 /$ j.ejso.2007.01.007 
9 Hollandsworth HM, Amirfakhri S, Filemoni F, Schmitt V, Wennemuth G, Schmidt A, Hoffman RM, Singer BB and Bouvet $\mathrm{M}$ : Anti-carcinoembryonic antigen-related cell adhesion molecule antibody for fluorescence visualization of primary colon cancer and metastases in patient-derived orthotopic xenograft mouse models. Oncotarget 11(4): 429-439, 2020. PMID: 32064046. DOI: 10.18632/oncotarget.27446

10 Iseki K, Tatsuta M, Iishi H, Sakai N, Yano H and Ishiguro S: Effectiveness of the near-infrared electronic endoscope for diagnosis of the depth of involvement of gastric cancers. Gastrointest Endosc 52(6): 755-762, 2000. PMID: 11115912. DOI: $10.1067 / \mathrm{mge} .2000 .110455$

11 Habibollahi P, Figueiredo JL, Heidari P, Dulak AM, Imamura Y, Bass AJ, Ogino S, Chan AT and Mahmood U: Optical imaging with a cathepsin B activated probe for the enhanced detection of esophageal adenocarcinoma by dual channel fluorescent upper GI endoscopy. Theranostics 2(2): 227-234, 2012. PMID: 22400064. DOI: $10.7150 /$ thno. 4088

12 Tjalma JJ, Garcia-Allende PB, Hartmans E, Terwisscha van Scheltinga AG, Boersma-van Ek W, Glatz J, Koch M, van Herwaarden YJ, Bisseling TM, Nagtegaal ID, Timmer-Bosscha H, Koornstra JJ, Karrenbeld A, Kleibeuker JH, van Dam GM, Ntziachristos V and Nagengast WB: Molecular fluorescence endoscopy targeting vascular endothelial growth factor A for improved colorectal polyp detection. J Nucl Med 57(3): 480485, 2016. PMID: 26678613. DOI: 10.2967/jnumed.115.166975

13 Yano S, Takehara K, Miwa S, Kishimoto H, Tazawa H, Urata Y, Kagawa $S$, Bouvet $M$, Fujiwara $T$ and Hoffman RM: Fluorescence-guided surgery of a highly-metastatic variant of human triple-negative breast cancer targeted with a cancer-specific GFP adenovirus prevents recurrence. Oncotarget 7(46): 7563575647, 2016. PMID: 27689331. DOI: 10.18632/oncotarget.12314
14 Yano S, Takehara K, Miwa S, Kishimoto H, Hiroshima Y, Murakami T, Urata Y, Kagawa S, Bouvet M, Fujiwara T and Hoffman RM: Improved resection and outcome of colon-cancer liver metastasis with fluorescence-guided surgery using in situ GFP labeling with a telomerase-dependent adenovirus in an orthotopic mouse model. PLoS One 11(2): e0148760, 2016. PMID: 26849435. DOI: 10.1371/journal.pone.0148760

15 Kishimoto H, Zhao M, Hayashi K, Urata Y, Tanaka N, Fujiwara $\mathrm{T}$, Penman $\mathrm{S}$ and Hoffman RM. In vivo internal tumor illumination by telomerase-dependent adenoviral GFP for precise surgical navigation. Proc Natl Acad Sci U S A 106(34):14514-7, 2009. PMID: 19706537. DOI: 10.1073/pnas.0906388106

16 Kishimoto H, Aki R, Urata Y, Bouvet M, Momiyama M, Tanaka N, Fujiwara T and Hoffman RM. Tumor-selective, adenoviralmediated GFP genetic labeling of human cancer in the live mouse reports future recurrence after resection. Cell Cycle 10(16):27372741, 2011. PMID: 21785265. DOI: 10.4161/cc.10.16.16756
Received April 5, 2021

Revised April 26, 2021

Accepted May 10, 2021 